hergestellter, gewebespezifischer Plasminogen-Aktivator - wird als Fibrinolytikum eingesetzt. Mehr als die Hälfte der Patienten mit einem akuten ischämischen Schlaganfall haben nur geringfügige neurologische Ausfälle. Zwar haben frühere größere Studien an Alteplase diese Patienten miteingeschlossen, Patienten ohne klare und behindernde Defizite wurden bisher aber nicht speziell untersucht.

\title{
Methoden
}

PRISMS ist eine doppelblinde, doppeltplacebokontrollierte, multizentrische Phase-Illb-Studie, in der 948 Patienten zwischen Mai 2014 und Dezember 2016 eingeschlossen wurden, um die Behandlung von Alteplase vs. Acetylsalicylsäure (ASS) bei akutem ischämischem Schlaganfall zu untersuchen. In der vorliegenden Studie wurden daraus 313 Patienten ausgewählt (mittleres Alter: 62; $46 \%$ Frauen) mit einem NIHSS-Score (National Institutes of Health Stroke Scale) von 0 bis 5 (mittlerer NIHSS-Score: 2). Alle Patienten wiesen nur leichte Symptome auf, beispielsweise eine Gesichtslähmung, Sensibilitäts- oder Sprachstörungen, und ihre Behandlung war innerhalb von 3 Stunden nach dem Ereignis begonnen worden (mittlere Zeit bis zur Behandlung: 2,7 h). Die Versuchspersonen erhielten randomisiert entweder intravenös Alteplase in der Standarddosis $(0,9 \mathrm{mg} / \mathrm{kg})$ mit einem oralen Placebo $(n=156)$ oder oral ASS $(325 \mathrm{mg})$ mit einem Placebo intravenös ( $n=157)$.

\section{Leichter Schlaganfall: Kein Vorteil von Alteplase gegenüber ASS}

Khatri P et al.: Effect of Alteplase vs Aspirin on Functional Outcome for Patients With Acute Ischemic Stroke and Minor Nondisabling Neurologic Deficits: The PRISMS Randomized Clinical Trial. JAMA. 2018; 320: 156-166. doi:10.1001/ jama.2018.8496

Die Arbeit evaluiert die Effizienz und Sicherheit von Alteplase bei Patienten mit nicht behindernden neurologischen Ausfällen. Alteplase-ein gentechnisch

\section{Ergebnisse}

Von den 313 Patienten haben 281 (89,8\%) die Studie abgeschlossen. Bezüglich des primären Studienendpunkts unterschieden sich die beiden Gruppen kaum: Nach 90 Tagen wiesen 122 Patienten (78,2\%) in der Alteplase-Gruppe gegenüber 128 Patienten (81,5\%) in der ASS-Gruppe eine gute funktionelle Ergebnisse auf (Adjusted Risk Difference, -1,1\%; $95 \%$ KI, $-9,4 \%$ bis 7,3\%). Fünf der mit Alteplase behandelten Patienten (3,2\%), aber keiner der mit ASS behandelten Patienten hatten eine symptomatische intrakranielle Blutung (Risk Difference, 3,3\%; $95 \% \mathrm{KI}, 0,8-7,4 \%$ ). 
FAZIT

Ob leichtgradig neurologisch beeinträchtigte Schlaganfallpatienten akut mit dem Fibrinolytikum oder mit ASS behandelt werden, scheint keinen Einfluss auf das Behandlungsergebnis nach 90 Tagen und den Schweregrad späterer Behinderungen zu haben. Durch den vorzeitigen Studienabbruch aufgrund der schleppenden Rekrutierung lassen sich jedoch die beiden Therapieregime nicht abschließend bewerten.

Dr. Markus Numberger, Kandel 